\title{
Cusped Mass Models Of Gravitational Lenses
}

\author{
J.A. Muñoz ${ }^{1}$, C.S. Kochanek ${ }^{2}$ and C.R. Keeton ${ }^{3}$ \\ ${ }^{1}$ Instituto de Astrofísica de Canarias, E-38200 La Laguna, Tenerife, Spain \\ email: jmunoz@ll.iac.es \\ ${ }^{2}$ Harvard-Smithsonian Center for Astrophysics, 60 Garden St., Cambridge, MA 02138 \\ email: ckochanek@cfa.harvard.edu \\ ${ }^{3}$ Steward Observatory, University of Arizona, Tucson, AZ 85721 \\ email: ckeeton@as.arizona.edu
}

\begin{abstract}
Recent observations of galaxy luminosity profiles and dark matter simulations find luminosity and mass distributions characterized by central cusps rather than finite core radii. We introduce and implement a set of cusped ellipsoidal lens models which include limits similar to the Jaffe, Hernquist, $\eta$ and NFW models and apply them to the gravitational lenses APM $08279+5255$ and B 1933+503. A successful model of APM $08279+5255$ with its central, odd image requires a very shallow cusp, $\gamma \lesssim 0.4$ where $\rho \propto r^{-\gamma}$ as $r \rightarrow 0$, which is similar to a core rather than the favored $1 \lesssim \gamma \lesssim 2$ cusps. B $1933+503$, by contrast, is well modeled with a steep density cusp, $1.6 \lesssim \gamma \lesssim 2.0$.
\end{abstract}

Subject headings: cosmology: gravitational lensing

\section{Introduction}

The families of density distributions used to model gravitational lenses are rooted in the lore of the early 1980s, where massive galaxies were believed to have finite core radii (e.g. Lauer 1985). Hence, surveys of lens models almost always use models related to the softened power-law surface density, $\Sigma \propto\left(m^{2}+s^{2}\right)^{(\alpha-2) / 2}$ where $m^{2}$ is a two-dimensional ellipsoidal coordinate and $s$ is the core radius, as a template for exploring the effects of the radial mass distribution on lensing phenomenon (e.g. Kochanek 1995, Chen, Kochanek \& Hewitt 1995, Grogin \& Narayan 1996, Chae, Turnshek \& Khersonsky 1998, Schmidt, Webster \& Lewis 1998, Keeton \& Kochanek 1998, Chae 1999, Keeton et al. 2000, Cohn et al. 2001). The asymptotic exponent $\alpha$ covers a range of historical models for galaxies from the singular isothermal ellipsoid (or SIE, $\alpha=1$ and $s=0$ ), to the modified Hubble profile $(\alpha=0)$ and the Plummer model $(\alpha=-2)$. Viewed as projections of thin disks, these models include the Mestel (1963) disk (equivalent to the SIE), and the Kuzmin (1956) disk $(\alpha=-1)$. For $\alpha=-1$ and 1 there are simple analytic expressions for the potentials 
(see Keeton \& Kochanek 1998 and references therein), and relatively fast approximation schemes have been developed for a broad range of exponents (Barkana 1998, Chae, Khersonsky \& Turnshek 1998). Unfortunately, the only model from this broad class of potentials that can be regarded as astrophysical is the SIE.

Since the advent of HST and high resolution numerical simulations, our view of the central regions of galaxies has changed significantly, in that massive galaxies have central cusps rather than central core radii in both high resolution observations of luminosity profiles (e.g. Faber et al. 1997) and theoretical simulations (e.g. Navarro, Frenk \& White 1997, Moore et al. 1998). Most simple, modern, analytic representations of galaxy mass distributions have density distributions of the form $\rho \propto r^{-\gamma}(r+a)^{-n+\gamma}$ where $\gamma$ is the exponent of the central density cusp, $n$ is the exponent for the asymptotic slope of the density distribution, and $a$ is the break radius. Popular limits are the Hernquist (1990) model $(\gamma=1, n=4)$, the Jaffe (1983) model $(\gamma=2, n=4)$, the more general $\eta$-models (Dehnen 1993, Tremaine et al. 1994, $n=4$ ), the Navarro, Frenk \& White (1997, NFW) models $(\gamma=1, n=3)$ and the more general Zhao (1996) models. The classical de Vaucouleurs (1948) profile for early-type galaxies is similar to the Hernquist model, although its central "cusp" is better approximated by a slightly shallower exponent (Hernquist 1990).

Few of these more realistic models have been considered as lens models. Lehar et al. (2000) fit SIE and ellipsoidal de Vaucouleurs models as standard models which represent the two typical limits of central cusps. Only one analytic study by Evans \& Wilkinson (1998) explored the general mathematics of models with different central cusps, focusing on circular models because there are no general analytic results for cusped ellipsoidal models. Bartelmann (1996) explored using the NFW density distribution to explain the radial arcs sometimes found in cluster lenses, and Wyithe, Turner \& Spergel (2000) and Keeton \& Madau (2001) explored the general statistical properties of a family of circular, cusped models for clusters. Cusps have qualitatively different lensing properties from finite cores, making it important to begin exploring their effects on gravitational lenses. In $\S 2$ we introduce a general class of ellipsoidal, cusped density distributions for use in gravitational lensing, and in $\S 3$ we apply the new models to the gravitational lenses APM $08279+5255$ and B $1933+503$. Our results are summarized in $\S 4$. The models are implemented and available as part of the lensmodel package of lens modeling codes (Keeton 2001)."

\section{A New Class of Ellipsoidal Lens Model}

Our models are defined from the spherical density distribution,

$$
\rho=\frac{\rho_{0}}{r^{\gamma}} \frac{a^{n}}{\left(r^{2}+a^{2}\right)^{(n-\gamma) / 2}}
$$

\footnotetext{
${ }^{1}$ The lensmodel package can be obtained from http://cfa-www.harvard.edu/castles
} 
for cusp exponent $\gamma$, break radius $a$ and outer exponent $n$ (it is the same as a Zhao (1996) model with $\alpha=1 / 2)$. This differs from the standard cusped models in using $\left(r^{2}+a^{2}\right)^{(n-\gamma) / 2}$ rather than $(r+a)^{n-\gamma}$, leading to a smoother break in the density profile, more convenient analytic properties for the lensing calculations, and overlaps with several standard lens models. This form also has physical advantages in some cases. For example, the rotation curve of the Jaffe (1983) model, $\rho \propto r^{-2}(r+a)^{-2}$, declines linearly for $r<a$ despite the $1 / r^{2}$ central density profile, while our "pseudo-Jaffe" model with $\rho \propto r^{-2}\left(r^{2}+a^{2}\right)^{-1}$ has a fairly flat rotation curve for $r<a$. Whenever the exponents of our density model match those of a standard cusped model we will refer to it as a "pseudo-" model. Hence for $n=4$, we have the pseudo-Jaffe model $(\gamma=2)$, the pseudo-Hernquist model $(\gamma=1)$, and the pseudo- $\eta$ models (any $\gamma$ ), and for $n=3$ and $\gamma=1$ we have the pseudo-NFW model. The model also matches the standard SIE model $(\gamma=2, n=4$ and $r \ll a$ ) and can match all the softened power-law models (for $\gamma=0$ and viewing the break radius $a$ as a core radius).

Given the density distribution, the total mass of the model is

$$
M_{T}=2 \pi a^{3} \rho_{0} B\left(\frac{n-3}{2}, \frac{3-\gamma}{2}\right)
$$

where $B(a, b)=\Gamma(a) \Gamma(b) / \Gamma(a+b)$ is the Euler beta function. The mass is well-defined (i.e. finite) only for $\gamma<3$ and $n>3$. The surface mass density in units of the critical surface density for lensing is

$$
\kappa(R)=\frac{\Sigma(R)}{\Sigma_{\text {crit }}}=\kappa_{0} B\left(\frac{n-1}{2}, \frac{1}{2}\right)\left(1+\xi^{2}\right)^{(1-n) / 2}{ }_{2} F_{1}\left(\frac{n-1}{2}, \frac{\gamma}{2}, \frac{n}{2} ; \frac{1}{1+\xi^{2}}\right)
$$

where the normalization $\kappa_{0}=\rho_{0} a / \Sigma_{\text {crit }},{ }_{2} F_{1}$ is the hypergeometric function and $\xi=R / a$ is the projected radius $R$ normalized by the break radius $a$. For a circular lens with $n \neq 3$, the deflection angle is

$$
\alpha(R)=\frac{2 \kappa_{0} a}{\xi}\left[B\left(\frac{n-3}{2}, \frac{3-\gamma}{2}\right)-B\left(\frac{n-3}{2}, \frac{3}{2}\right)\left(1+\xi^{2}\right)^{(3-n) / 2}{ }_{2} F_{1}\left(\frac{n-3}{2}, \frac{\gamma}{2}, \frac{n}{2} ; \frac{1}{1+\xi^{2}}\right)\right]
$$

For $n=3$ the deflection must be expressed in terms of generalized hypergeometric functions. The first derivative of $\kappa$, which is used in some of the integrals needed to compute the properties of ellipsoidal models, is

$$
\begin{aligned}
\frac{d \kappa(R)}{d R}= & -\frac{\kappa_{0}}{a} B\left(\frac{n+1}{2}, \frac{1}{2}\right) \xi\left(1+\xi^{2}\right)^{-(n+3) / 2}\left[n\left(1+\xi^{2}\right){ }_{2} F_{1}\left(\frac{n-1}{2}, \frac{\gamma}{2}, \frac{n}{2} ; \frac{1}{1+\xi^{2}}\right)+\right. \\
& \left.+\gamma_{2} F_{1}\left(\frac{n+1}{2}, \frac{\gamma+2}{2}, \frac{n+2}{2} ; \frac{1}{1+\xi^{2}}\right)\right]
\end{aligned}
$$

In Figure 1 we plot the deflection angle, $\alpha(R)$, for several values of $\gamma$, a fixed outer exponent of $n=4$ and a fixed surface density scale $M_{T} / \pi a^{2} \Sigma_{c r i t}=1$. The central deflection diverges for $\gamma>2$, is finite for $\gamma=2$ and passes through zero for $\gamma<2$. 


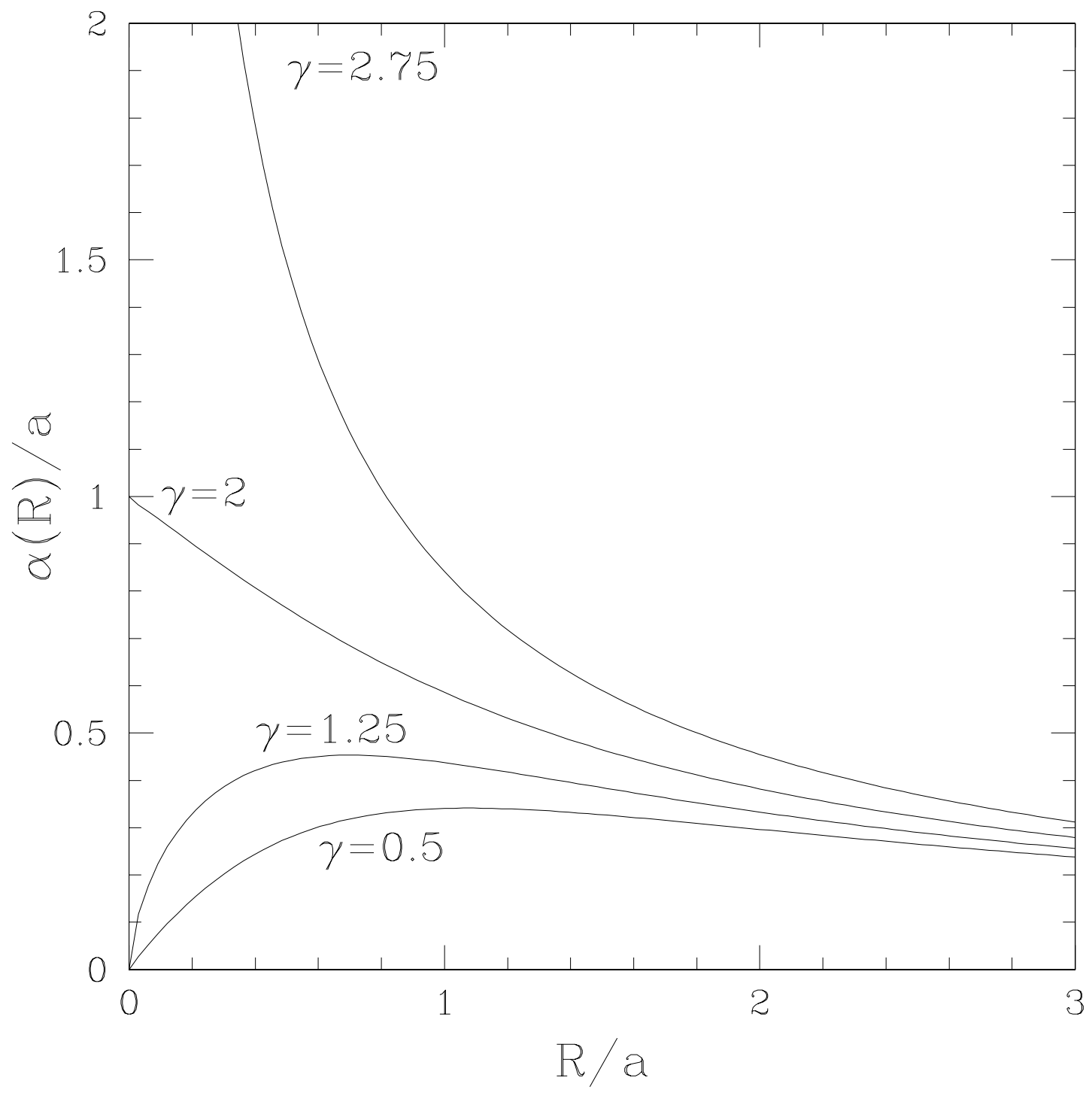

Fig. 1. - The deflection angles of cusped models for several values of $\gamma$, where the central density $\rho \propto r^{-\gamma}$, for a fixed outer exponent of $n=4$ and a mass normalization of $M_{T} / \pi a^{2} \Sigma_{c r i t}=1$. 
The properties of the lens models in the core can be better understood by examining power series expansions of the deflection angle and the magnification as $\xi=R / a \rightarrow 0$. If we define the constants $A \equiv \kappa_{0} B[(n-1) / 2,(1-\gamma) / 2]$ and $B \equiv \kappa_{0} B[(\gamma-1) / 2,1 / 2]$, for $\gamma \neq 1$ we find that

$$
\begin{gathered}
\kappa(R)=A+B \xi^{1-\gamma}+O\left(\xi^{2}, \xi^{3-\gamma}\right) \\
\alpha(R)=R\left[A+\frac{2 B}{3-\gamma} \xi^{1-\gamma}+O\left(\xi^{2}, \xi^{3-\gamma}\right)\right]
\end{gathered}
$$

with logarithmic divergences when $\gamma=1$. For circular lenses, the inverse of the magnification, $\mu^{-1}=1-2 \kappa(R)-\alpha^{2}(R) / R^{2}+2 \kappa(R) \alpha(R) / R$, can be expressed in terms of $\kappa(R)$ and $\alpha(R)$ (e.g. Schneider, Ehlers and Falco 1992). Using eqns. (6) and (7), we find a power series expansion for the central magnification of

$$
\mu^{-1}=(A-1)\left(A-1+2 B \xi^{1-\gamma}\right)+O\left(\xi^{2(1-\gamma)}, \xi^{2}, \xi^{3-\gamma}\right)
$$

For $\gamma \geq 2$ the cusp model produces only an even number of images; there is no central "odd" image. For $\gamma<2$ the model does produce a central, odd image, but for $1<\gamma<2$ the image can be significantly demagnified compared to the other images (e.g. Evans \& Wilkinson 1998).

When we make the models ellipsoidal we must calculate the deflections and magnifications numerically. The projected radius $R$ is replaced by the ellipsoidal coordinate $R^{2} \rightarrow \eta^{2}=x^{2}+y^{2} / q^{2}$ where $q$ is the projected axis ratio and the Cartesian coordinates $x$ and $y$ are rotated to match the principal axes of the lens. The elliptical symmetry reduces the computation of the lensing properties to one-dimensional integrals (see e.g. Schramm 1990). Modeling lenses using the numerical integrals is too slow for practical use, so we tabulate the potential, the deflections and the components of the magnification tensor as a function of scaled radius $(x / a$ and $y / a)$ and axis ratio $(q)$. The modeling is then done by interpolating over the tabulated models. We have implemented the ellipsoidal cusped models as part of the lensmodel software package (Keeton 2001).

\section{Application of the New Model to APM $08279+5255$ and B $1933+503$}

We now apply these models to two gravitational lenses. The first lens, APM 08279+5255 (Irwin et al. 1998), is the first clear example of a gravitational lens with a bright central image (see Ibata et al. 1999, Egami et al. 2000). In this system we can explore the role of a shallow central cusp, rather than a finite core radius, in producing a visible central image. The second lens, B 1933+507 (Sykes et al. 1998), is a 10 image gravitational lens which is well-suited to determining the radial mass distribution of the lens galaxy. A detailed analysis by Cohn et al. (2001) found that models with cusps slightly shallower than isothermal produced the best fits, but these models were not globally consistent due to their infinite masses and perpetually rising rotation curves. 


\subsection{APM $08279+5255$}

When Irwin et al. (1998) discovered the $z_{s}=3.87$ BAL quasar APM 08279+5255 they also noted that the image was extended and likely to be a compact double (confirmed by Ledoux et al. 1998). HST images in the infrared with NICMOS (Ibata et al. 1999) and improved ground-based images (Egami et al. 2000) demonstrated that the source was actually a triple. There are two bright point sources separated by 0 '! 38 with a flux ratio of 0.8 , and between the two lies a relatively bright third image with a flux ratio of 0.2 relative to the brightest image. A lens galaxy has yet to be detected, although this is not surprising given the extraordinary brightness of the quasar images (about $15 \mathrm{R}$ mag!). The image configuration is easily modeled as an isothermal ellipsoid with a finite core radius (Ibata et al. 1999, Egami et al. 2000). However, given our expectation that the lens galaxy should not have a core radius, we will investigate whether the system is equally well modeled by a shallow cusp.

While it is likely that the system is composed of three lensed images given the fluxes and the similarity of the infrared colors (see Ibata et al. 1999), we can use optical flux ratios for further confirmation. The CfA/Arizona Space Telescope Lens Survey (CASTLES) obtained I-band (F814W) and V-band (F505W) images of APM 08279+5255 on 1999.11.09 with the Hubble Space Telescope (HST). Each observation consisted of 20 dithered $40 \mathrm{sec}$ exposures designed to avoid saturating the extraordinarily bright quasars. The data were reduced and analyzed following the standard procedures of Lehar et al. (2000) for the CASTLES observations. Table 1 presents the resulting astrometry and fluxes for the system where we also include the photometry obtained from Ibata et al. (1999) with the NICMOS infrared images. We now see that not only are the infrared colors of the images similar, but the spectrum remains similar over the full range from the V-band to K-band, which makes it virtually impossible for the three images to be anything other than three images of the BAL quasar. We use these results to constrain our lens models with a more conservative error of 0. ! 01 for the quasar positions.

We first fit the system using a softened isothermal ellipsoid. Like Ibata et al. (1999) and Egami et al. (2000) we find that the model produces a perfect fit to the data given a core radius of $a=00^{\prime \prime} 16_{-0.0_{05}^{+\prime}, 10}$, which would be $0.7_{-0.2}^{+0.4}\left(0.9_{-0.3}^{+0.6}\right) h^{-1} \mathrm{kpc}$ for a lens redshift of $z_{l}=0.5(1.0)$ in an $\Omega_{0}=0.3$ flat cosmological model. Since the bulges of massive galaxies usually show cusps down to smaller scales (see e.g. Faber et al. 1997), this solution appears to be inconsistent with the known properties of galaxies. The best model reaches $\chi^{2}=0$, as we would expect given a system with 6 constraints and 6 parameters leaving $N_{d o f}=0$ degrees of freedom. We also fit a model where the lens was an exponential disk without a bulge or dark matter halo. Exponential disks have little density contrast between their centers and the Einstein ring, which makes it relatively easy to fit the system. We show the parameters of these two models in Table 2. Lens models with a de Vaucouleurs profile were not able to fit the data for any effective radius. The central density of the $R^{1 / 4}$ law roughly corresponds to a central density distribution with $\rho \sim r^{-3 / 4}$ as $r \rightarrow 0$ (see Hernquist 1990), and a central exponent $\gamma=3 / 4$ is too steep to reproduce the central bright 


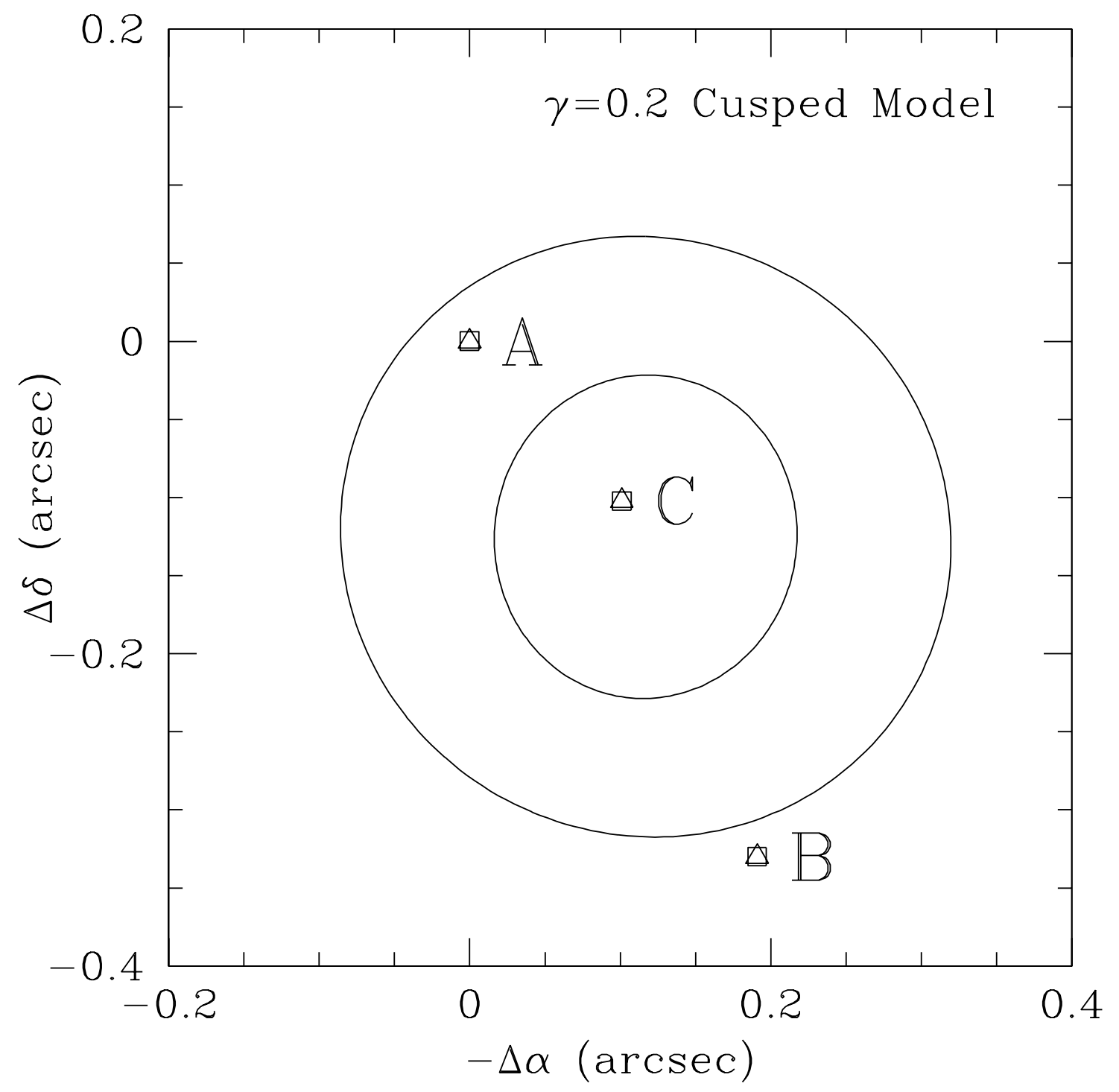

Fig. 2.- A schematic diagram of APM $08279+5255$. The squares show the observed positions of the three quasar images, and the triangles show the model positions in the best fitting $\gamma=0.2$ cusped model from Table 2. The inner and outer curves are the radial and tangential critical lines of the model. 


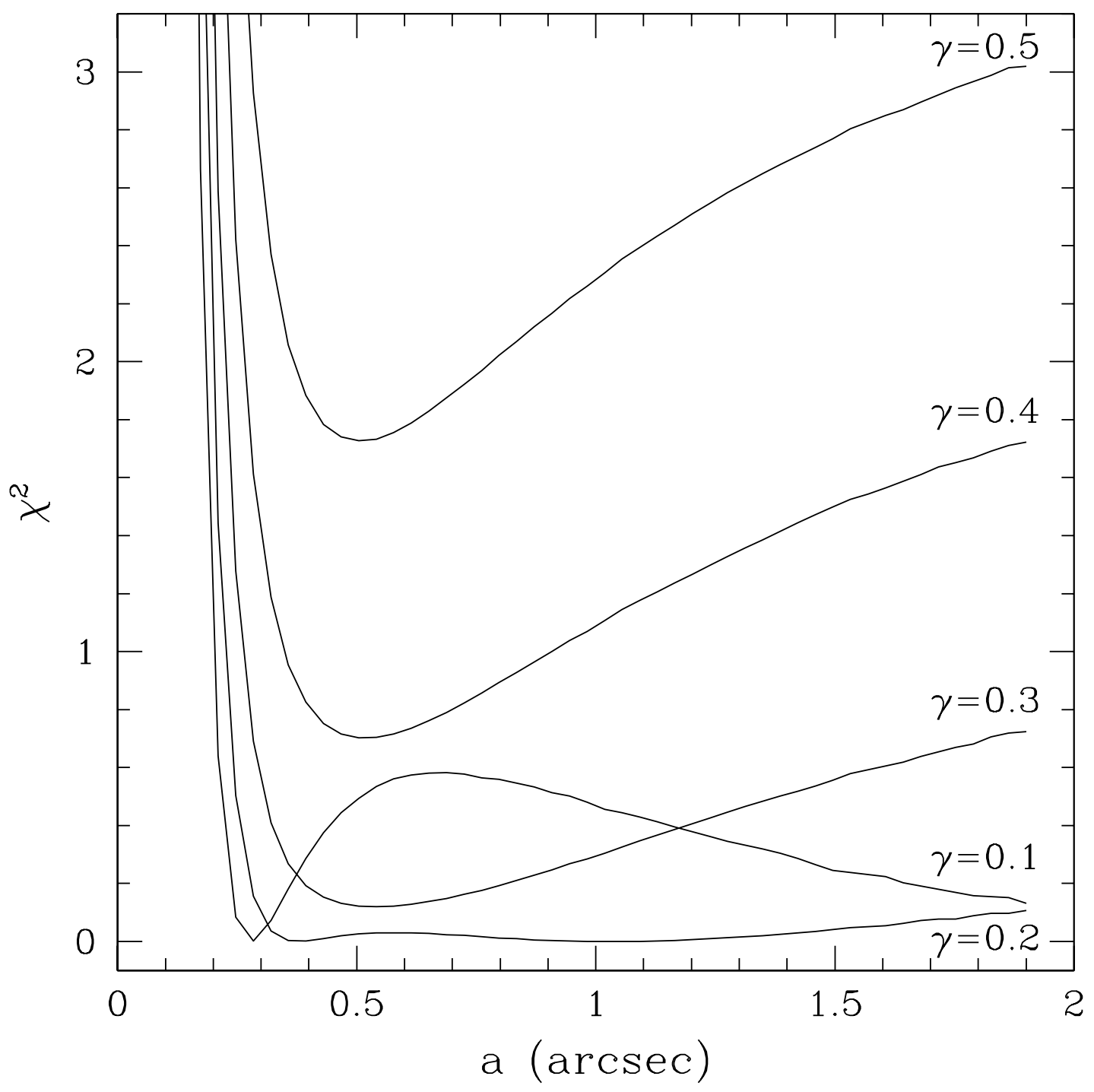

Fig. 3.- Ellipsoidal cusped models for APM 08279+5255. We show the $\chi^{2}$ statistic for the lens models as a function of the break radius $a$ for several values of the central cusp exponent $\gamma$. Note the presence of high (large $a$ ) and low (small $a$ ) magnification solution branches for $\gamma=0.1$ and 0.2. 
image (see below).

Next we fit a sequence of our cusped models to the system (see Figures $2 \& 3$ ). The models are underconstrained when we allow the cusp exponent and the break radius both to vary ( $N_{\text {dof }}=-1$ given 6 constraints and 7 parameters). Nevertheless, we could only find successful models for cusps outside the standard range of $1 \lesssim \gamma \lesssim 2$ found for stellar luminosity profiles and dark matter simulations. We found that the cusp exponent has to satisfy $\gamma \lesssim 0.4$ to achieve $\chi^{2}<1$, and the models can produce perfect fits only for $\gamma \lesssim 0.2$. These cusps are so shallow that we are almost at the limit of reproducing the softened power law models $(\gamma=0)$. While the cusp models with a small break radius have total magnifications comparable to the softened isothermal models $\left(\mu_{T} \simeq 100\right)$, there is a second solution branch with a very large break radius where the total magnification slowly diverges. For example a model with $\gamma=0.2$ and $a \sim 1^{\prime \prime}$ (see in Fig. 3) provides a perfect fit combined with a total magnification of $\mu_{T} \sim 1800$. Without any correction

for lensing, the implied luminosity of the system is $\sim 5 \times 10^{15} L_{\odot}(\Omega=1$, Egami et al. 2000), and in the previous models and most of our new models the lens magnification of $\mu_{T} \sim 100$ reduces this to a more physically plausible $\sim 5 \times 10^{13} L_{\odot}$. On the branch of solutions with $\mu_{T} \sim 1800$, however, the intrinsic luminosity of the system drops to $\sim 3 \times 10^{12} L_{\odot}$ and the source ceases to be remarkable in its intrinsic properties. We can find models with steeper central cusps $(1<\gamma<2)$ by adding an external shear, but these models are very underconstrained ( 9 parameters for 6 constraints) and require very high lens ellipticities. They are probably unphysical, and we do not present any examples.

Table 2 summarizes a representative set of models. Since the models are underconstrained and do not include an external shear (which we know is required in a complete model, see Keeton, Kochanek \& Seljak 1997), we do not include error estimates for the parameters. It is impossible to distinguish between the models using the current constraints, in large part because the actual deflection profiles of the models are very similar (see Fig. 4). The position of the lens galaxy is predicted to vary by only a few milli-arcseconds between the models, and it will be extraordinarily difficult to detect the lens galaxy at all given the enormous flux from the lensed images. With the exception of the high magnification models, the time delays vary by only $20 \%$ and a precise knowledge of the Hubble constant would be required to distinguish between the models. The delay ratios, which are independent of the Hubble constant, vary by only $1 \%$ between the models. The only prospect for significantly improving the constraints in this system is to search for the lensed images of the quasar host galaxy (see Kochanek, Keeton \& McLeod 2001), although the brightness of the quasar images makes this a challenging observation.

\subsection{B $1933+503$}

B 1933+507 (Sykes et al. 1998, Nair 1998, Marlow et al. 1999, Browne et al. 1999, Biggs et al. 2000, Norbury et al. 2000) has 10 lensed images of a $z_{s}=2.62$ radio source generated by a $z_{l}=0.76$ lens galaxy. The source has three radio components, two of which have 4 lensed images 


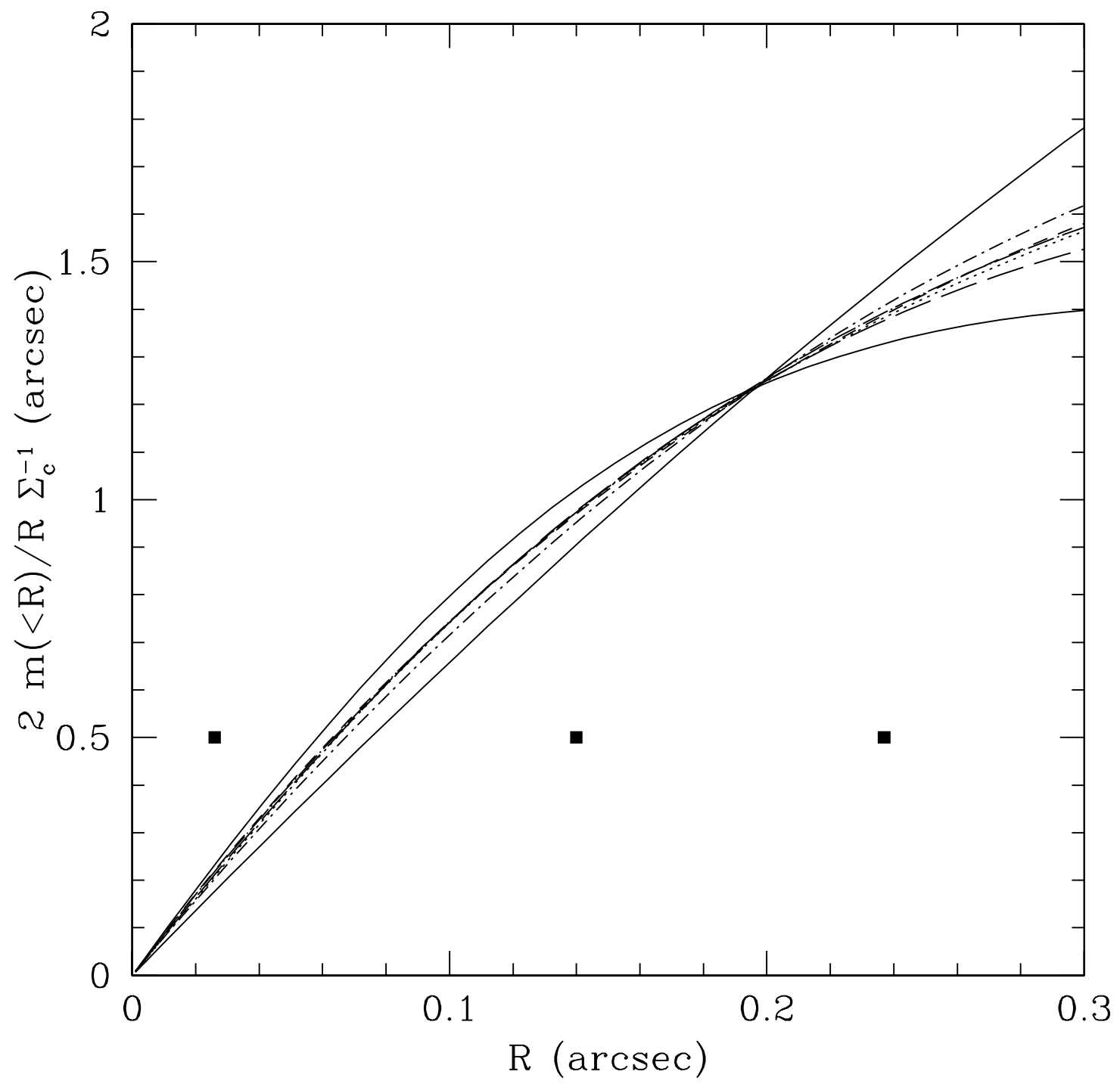

Fig. 4. - Monopole deflections for the lens models of APM 08279+5255 listed in Table 2. At $\mathrm{r}=0^{\prime \prime} \cdot 3$ the smaller (larger) value corresponds to the $\gamma=0.1\left(\gamma=0.2, a=11^{\prime \prime} 02\right)$ cusped model. The rest of the models (SIE with a finite core (dotted), exponential disk (short dashed) and $\gamma=0.2$ (long dashed), 0.3 (dot-short dashed), 0.4 (dot-long dashed) cusped models) are very similar at the image positions (marked by the solid points). In particular the softened SIE, exponential disk and $\gamma=0.4$ cusped models are almost indistinguishable. 
and one of which has two lensed images (see Fig. 5). The large number of constraints provided by the 10 images makes this lens an ideal laboratory for studying the radial mass distribution of the lens galaxy. Extensive models by Cohn et al. (2001) found that the best mass distribution they considered was a power law model with a singular core and an exponent slightly shallower than isothermal ( $s=0$ and $\alpha \simeq 1.1$ ). The $\alpha \gtrsim 1$ power-law models with zero core radius favored by the Cohn et al. (2001) analysis correspond to our cusped models with $\gamma=3-\alpha$ in the limit of infinite break radius, $a \rightarrow \infty$. The Cohn et al. (2001) models are unphysical because they have infinite mass and a rising rotation curve. Our new cusp models allow us to examine these solutions using the same central cusp exponents combined with an outer break radius to produce a physical model. We used the same constraints as Cohn et al. (2001) to study the system.

Figure 6 shows the goodness of fit as a function of the break radius for a representative range $(1.1 \leq \gamma \leq 2.1)$ of cusp exponents. Cusps shallower than $\gamma=1.1$ are ruled out because they produce observable central images. The best fit models for a range of cusp exponents are presented in Table 3, and Figure 7 shows goodness of fit and the permitted range of break radii as a function of the cusp exponent. The best fit model has $\gamma=1.85$ and a large break radius (which we fixed to $a=10^{\prime \prime}$ ) with a total fit statistic $\chi^{2}=10.8$ for 17 degrees of freedom given that the cusped model has 9 parameters and the data supplies 26 constraints (see Cohn et al. 2001). Figure 5 compares the data and the model images and shows the locations of the source components relative to the model caustics. The largest contribution to the $\chi^{2}(4.3)$ is due to the position of image 1a (see Fig. 5). This best fit model is nearly identical to that of Cohn et al. (2001) who considered models with the same central density exponent but an infinite break radius.

The cusp exponent is limited to the range $1.6 \lesssim \gamma \lesssim 2.0$, marginally excluding the isothermal exponent $\gamma=2$ and strongly excluding the popular $\gamma=1$ cusps of the NFW and Hernquist models. Shallower cusps, $\gamma<1.8$, require finite break radii that become steadily smaller as the cusp becomes shallower. In other words, to maintain a large enough surface density contrast between the center of the lens and the Einstein ring to avoid creating visible central images, the shallower cusps require global profiles that rapidly break onto the steeper outer profile. As pointed out by Rusin \& Ma (2000), shallow cusps of infinite extent $(\gamma<1.8$ with $a \rightarrow \infty)$ can be ruled out because they will produce visible central images in too many lensed systems to be consistent with the data. Here, we can rule out the shallower cusps for any break radius, rather than the limiting case of a break radius which is large compared to the Einstein ring of the lens. Steeper central cusps, $\gamma>2$, intrinsically lack central images (see §2), but are also inconsistent with the constraints.

Figure 8 shows the monopole deflection of lens models which are consistent with the data. Despite the enormous freedom in the models, only models with nearly flat rotation curves are consistent with the data. The dashed and solid lines are used to distinguish between the models with $\gamma<1.8$ where the best lens model has a finite break radius and $\gamma \geq 1.8$ where the best model is reached for $a \rightarrow \infty$. This discontinuity produces two different trends in the monopole deflection as well as a small shift in the crossing point. 

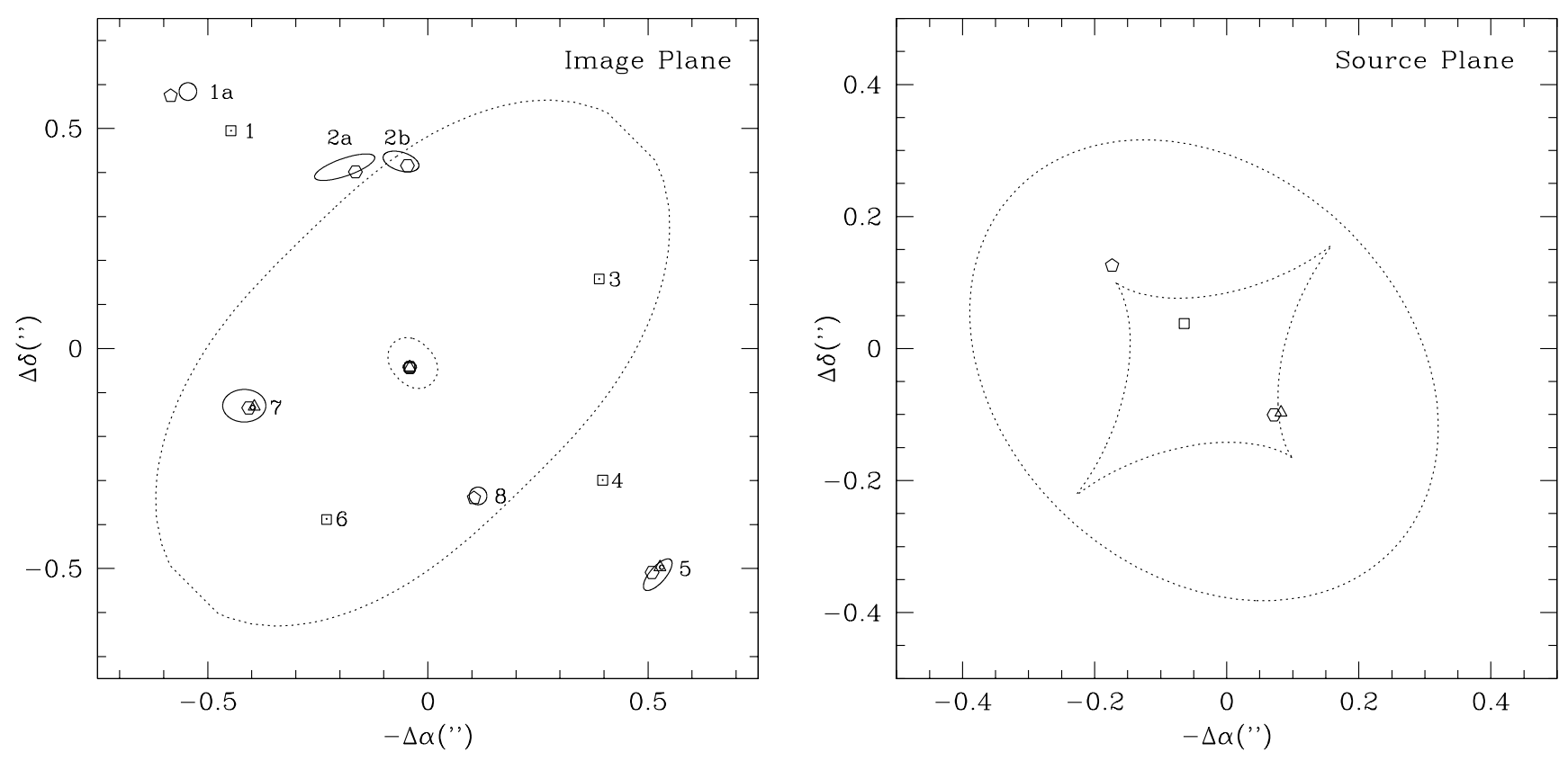

Fig. 5.- Schematic diagrams of the image (left) and source (right) planes of B $1933+503$. The lensed image is composed of 3 source components. The compact core is lensed into 4 images ( 1 , 3,4 and 6) marked with squares in the left panel. The first steeper spectrum source component is lensed into 2 images $(1 \mathrm{a}, 8)$ marked with pentagons. The second steeper spectrum source component straddles a caustic and is lensed into a combination of two images ( 5 and 7 ) marked with triangles and four images $(2 \mathrm{a}, 2 \mathrm{~b}, 5$ and 7$)$ marked with hexagons. The error ellipses on the image plane show the observed image positions and their uncertainties. The points are the model image and source positions for the $\gamma=1.85$ cusp model from Table 2. The inner (outer) dashed lines on the image plane show the radial and tangential critical lines, while the outer (inner) dashed lines on the source plane show the radial and tangential caustics. 


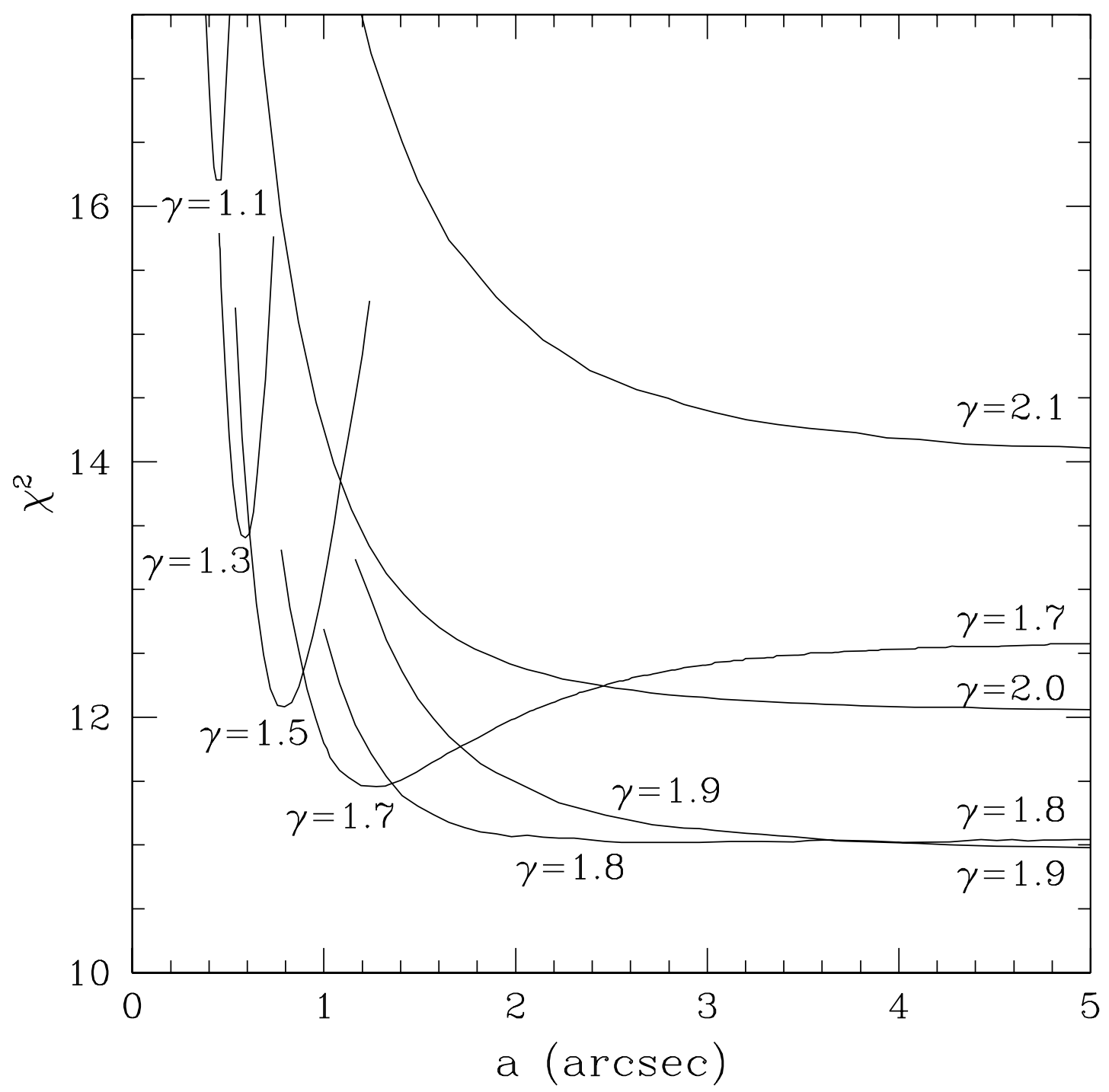

Fig. 6.- Model $\chi^{2}$ statistics for B $1933+503$ as a function of the break radius $a$ for a range of cusp exponents, $\gamma$. The lens model, consisting of an ellipsoidal cusp in an external shear field, has $N_{\text {dof }}=17$ degrees of freedom. 

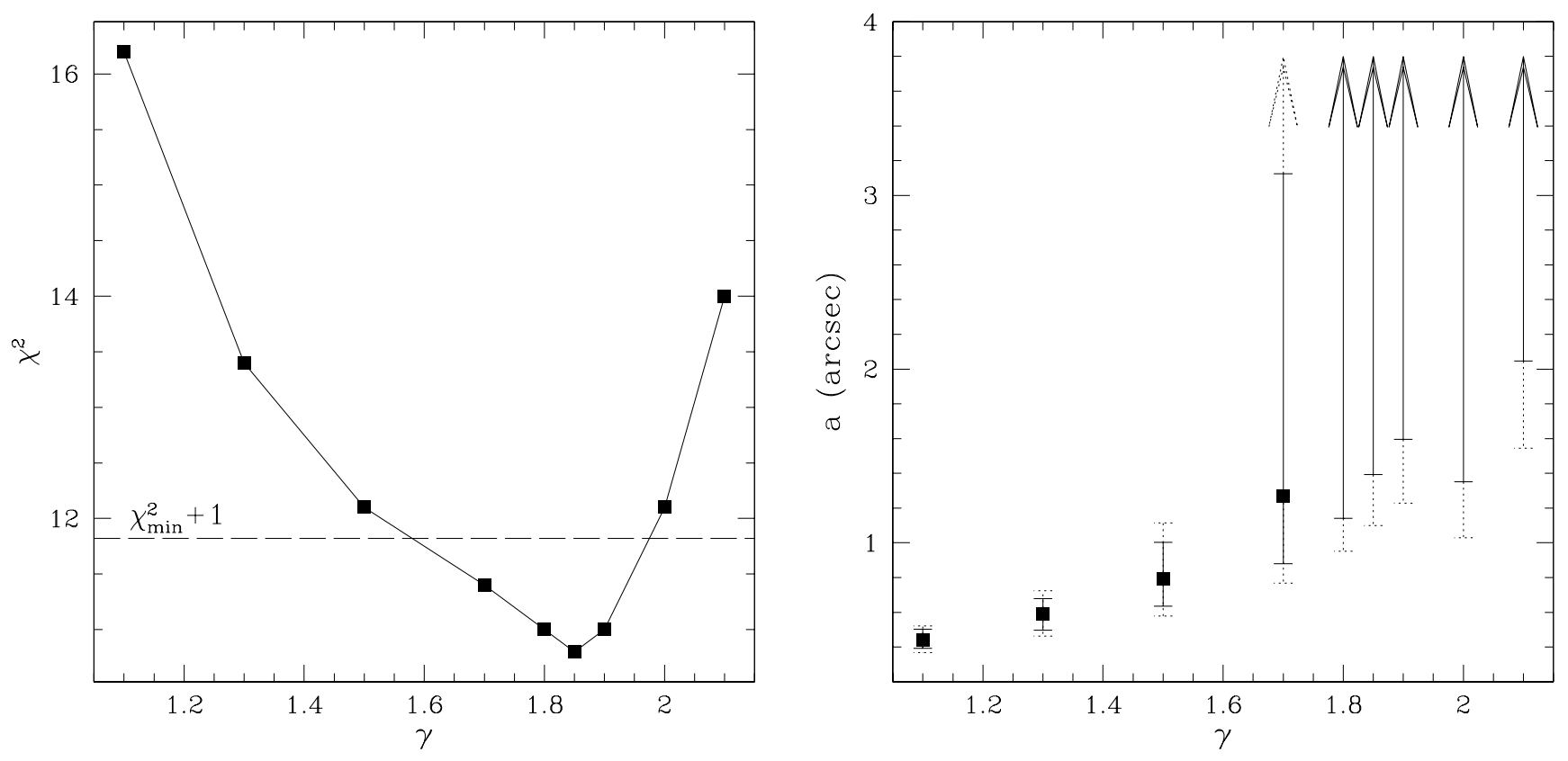

Fig. 7.- (Left) The minimum $\chi^{2}$ statistic for B $1933+503$ as a function of the cusp exponent $\gamma$ after optimizing the break radius. (Right) The optimal, 1- $\sigma$ and $2-\sigma$ range limits in B 1933+503 for the break radius $a$ as a function of the cusp exponent $\gamma$. The limits were determined by the $\Delta \chi^{2}=1$ range (solid lines) and the $\Delta \chi^{2}=2$ (dashed lines) from the minimum $\chi^{2}$ (points) found for each value of the cusp exponent. For $\gamma \geq 1.8$ the minimum $\chi^{2}$ is reached when $a \rightarrow \infty$. 


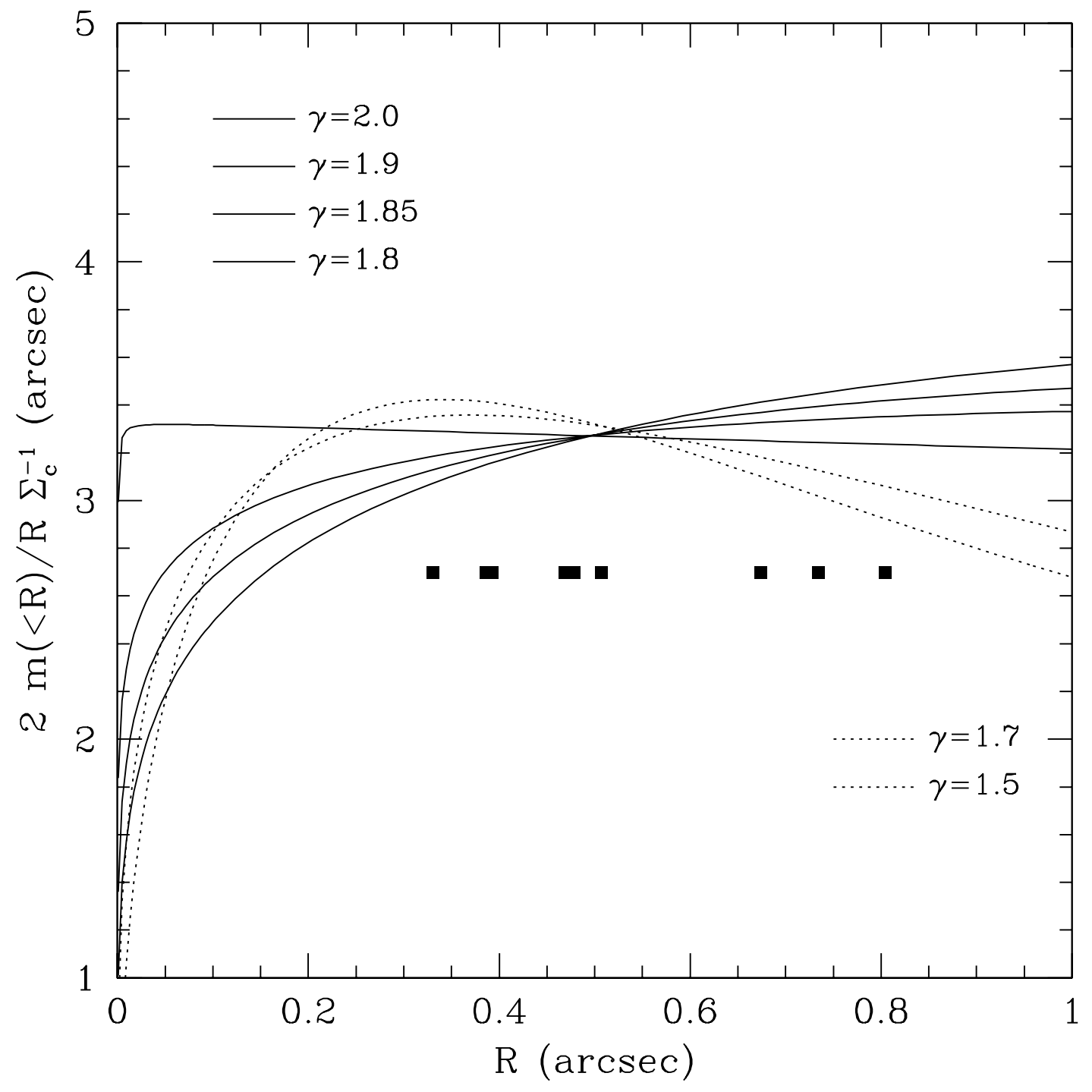

Fig. 8. - The monopole deflection of the cusped models for B 1933+503. The solid (dotted) lines correspond at $r=0\left(1^{\prime \prime}\right)$ from bottom to top with cusp exponents $\gamma=1.8,1.85,1.9,2.0(1.5,1.7)$. The monopole deflection is roughly proportional to the square of the rotation curve, $v_{c}(r)^{2}$. The points show the radial positions of the lensed images. 


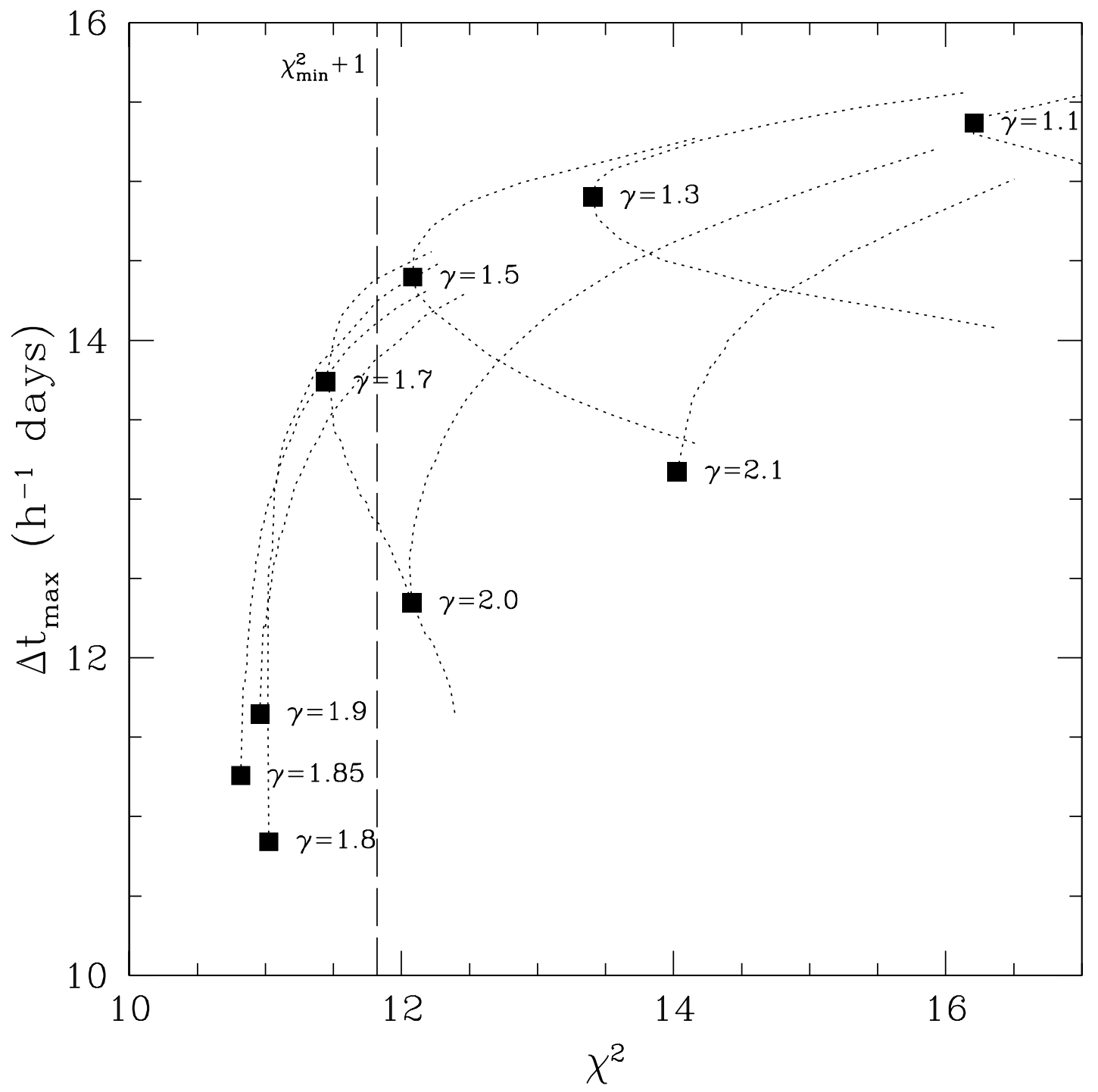

Fig. 9.- Time delay predictions for B $1933+503$ as a function of the $\chi^{2}$. We display only the longest time delay between the four images of the compact radio-core (it corresponds to images 1 and 6, see Figure 5) given in days in a flat $\left(\Omega_{0}=0.3\right)$ cosmology with $H_{0}=100 \mathrm{~h} \mathrm{~km} \mathrm{~s}^{-1} \mathrm{Mpc}^{-1}$. The points correspond to the best lens models for different values of $\gamma$. The dotted lines show the variation of the time delay as a function of the $\chi^{2}$ when we vary the break radius for each value of $\gamma$. The dashed line shows the limit $\Delta \chi^{2} \leq 1$. For $\gamma \geq 1.8$ the solid points correspond to break radius of $10^{\prime \prime} 0$, and further increases in the break radius do not significantly reduce the delays (for $\gamma=2$, the delay decreases by only $2.5 \%$ as the break radius rises from $a=10^{\prime \prime} 0$ to $a=\infty$ ). 
The location of the outer break radius does affect the time delays between the images, where for a given $\gamma$ we find shorter delays as we increase the break radius. Figure 9 shows the longest time delay between the four images of the compact radio-core (it corresponds to images 1 and 6 , see Figure 5) as a function of the $\chi^{2}$. The points correspond to the best lens models for different values of $\gamma$. The dotted lines show the variation of the time delay as a function of the $\chi^{2}$ when we vary the break radius for each value of $\gamma$. The dashed line shows the limit $\Delta \chi^{2} \leq 1$. Given the generality of the cusped model we can consider the values inside this region as a very robust prediction of the time delay. This figure also shows more clearly the consequences of the jump in the optimal break radii from finite to infinite at $\gamma \simeq 1.8$.

\section{Summary}

Gravitational lenses are used both to estimate the mass distribution of galaxies (e.g. Kochanek et al. 1989, Kochanek 1991, Kochanek \& Narayan 1992, Wambsganss \& Paczynski 1994, Kochanek 1995, Chen et al. 1995, Wallington, Kochanek \& Narayan 1996, Ellithorpe, Kochanek, \& Hewitt 1996, Chae et al. 1998, Schmidt et al. 1998, Kochanek et al. 2000, Cohn et al. 2001) and the Hubble constant from measurements of the time delays between the lensed images (e.g. Grogin \& Narayan 1996, Kundic et al. 1997, Schechter et al. 1997, Keeton \& Kochanek 1997, Impey et al. 1998, Chae 1999, Barkana et al. 1999, Koopmans \& Fassnacht 1999, Fassnacht et al. 1999, Keeton et al. 2000, Kochanek, Keeton \& McLeod 2001, Oscoz et al. 2001). Almost all these analyses use parametric models for the mass distribution of the lens. Parametric models have three advantages: first, all standard parametric models represent physically self-consistent, dynamically stable equilibrium systems; second, parametric models are relatively easy to interpret physically; and third, they are computationally efficient. The disadvantage of parametric models, particularly for estimating the systematic uncertainties in the mass distribution or the Hubble constant, is that they may overly restrict the true freedom in the mass distributions of real astronomical systems.

Our new family of ellipsoidal models for gravitational lenses is a significant advance for two reasons. First, the new models are far more realistic models of galaxies than the standard lens models given our current understanding of the central stellar density profiles of galaxies (e.g. Faber et al. 1997) and simulations of dark matter halos (Navarro et al. 1997, Moore et al. 1998), where the central density follows a power-law cusp with $\rho \propto r^{-\gamma}$ and $1 \lesssim \gamma \lesssim 2$. Our model, which is a subcase of the more general Zhao (1996) models, includes analogues of many currently popular analytic density distributions such as the Jaffe (1983) model, the Hernquist (1990) model, the $\eta$ model (Dehnen 1993, Tremain et al. 1994) and the NFW model (Navarro et al. 1997). Second, independent of the models being physically more realistic than the standard lens models, they provide a genuinely different class of density distributions from the standard lens models. Since the freedom in the radial mass distribution appears to be the dominant source of systematic uncertainties in determining the Hubble constant from gravitational lenses (see e.g Impey et al. 1998, Fassnacht et al. 1999, Keeton et al. 2000), the new models can be used as an additional 
probe of the uncertainties. The cusped models are implemented and publically available as part of the lensmodel (Keeton 2001) package for modeling gravitational lenses.

Non-parametric models of the mass distribution avoid the restrictions of parametric models. In particular, the elegant linear programming method introduced by Saha \& Williams (1997) assumes only that the surface mass density of the lens is non-negative. This method will find a broader range of systematic uncertainties in the mass distribution or the Hubble constant than any parametric model or set of parametric models. Unfortunately, the models also lose the first advantage of the parametric models, that they correspond to physical, dynamically stable systems. Physical systems must have non-negative density distributions and phase space distribution functions, and many non-negative surface densities will correspond to unphysical models with regions of negative density or phase space density (see Cohn et al. 2001). Models which are physical may still be improbable because they are dynamically unstable, with lifetimes of only a few dynamical (crossing) times.2 These additional criteria mean that the current non-parametric methods greatly over-estimate the freedom in the mass distribution and frequently produce unphysical models. These issues make the two approaches to modeling lenses complementary. The parametric models are always physical but can underestimate the freedom in the mass distribution, while the non-parametric models are frequently unphysical and overestimate the freedom in the mass distribution.

We explored these new parameterized mass distributions by using them to model two lenses, APM 08279+5255 and B 1933+503. APM 08279+5255 (Irwin et al. 1998, Ibata et al. 1999, Egami et al. 2000) is the only lens which clearly possesses three images of the background source. We find further evidence of this from the CASTLES V-band and I-band HST images of the system, which show that the flux ratios of the images change little from the K-band in the infrared to the V-band in the optical. Although the ability of the cusped models to model a three image gravitational lens like APM $08279+5255$ without a finite core radius was one of the motivations for exploring the cusped models, we actually found that APM $08279+5255$ cannot be modeled by cusps in the favored range $1 \lesssim \gamma \lesssim 2$. Only models with $\gamma \lesssim 0.4$, where the cusps become

\footnotetext{
${ }^{2}$ It is possible to detail these additional conditions on the surface density $\Sigma(R)$ for spherical systems (see Binney $\&$ Tremaine 1987). The mass density, $\rho=-\pi^{-1} \int_{r}^{\infty} d R(d \Sigma / d R)\left(R^{2}-r^{2}\right)^{-1 / 2}$, must be positive for all $r$, which is trivially satisfied if the surface density distribution is a monotonically declining function, $d \Sigma / d R<0$. For an isotropic distribution function, which depends only on the binding energy $\epsilon$, the distribution function corresponding to a given density distribution is positive if $\int_{0}^{\epsilon}(d \rho / d \psi)(\epsilon-\psi)^{-1 / 2} d \psi$ is an increasing function of $\epsilon=\psi-v^{2} / 2$. This is trivially satisfied if $d \rho / d \psi>0$, which is true for all the parametric models. The system is dynamically stable if the derivative of the distribution function $d f / d \epsilon<0$. Each step in this process imposes a smoothness criterion (through the constraints on the derivatives of the previous variable) which goes well beyond simply requiring a positive surface density. Viewed in terms of the surface density $\Sigma$, a positive density is a restriction on $d \Sigma / d R$, a positive distribution function is a restriction on $d^{2} \Sigma / d R^{2}$, and stability is a restriction on $d^{3} \Sigma / d R^{3}$ ! Unfortunately, there are no analytic expressions even for spherical systems with distribution functions depending on energy and angular momentum, let alone systems with more complicated symmetries. Although galaxies are more complicated than simple spherical systems, the problems we illustrate using the spherical systems apply to all galaxies and mean that a positive surface density is only a first, minimal criterion for a physical mass model.
} 
very similar to finite core radii $(\gamma=0)$, could successfully explain the properties of this lens. This suggests that the lens galaxy in APM $08279+5255$ is a spiral galaxy, where rotation curves are sometimes consistent with finite central densities rather than steep central density cusps. Alternatively, this could be an example of a "disk" geometry lens (Keeton \& Kochanek 1998) where we see three images produced by a nearly edge on disk galaxy. In a disk lens geometry, the lens galaxy should be offset perpendicular to the A-B image separation. The small image separation (0'38) also argues for a spiral lens (see Fukugita \& Turner 1991, Kochanek 1996), but the similar colors of the three images suggests that there is little dust along the three lines of sight through the lens. Unfortunately, the extreme brightness of the lensed images makes it nearly impossible to find the far fainter lens galaxy, let alone measure its position and morphology.

The second lens we considered, B 1933+503 (Sykes et al. 1998), already had an extensive model survey suggesting that the best models would have steep central cusps (Cohn et al. 2001). Here we show using physically realistic cusped models rather than simple power law density distributions that the system requires a steep central cusp, $\rho \simeq r^{-1.8 \pm 0.2}$, with a break radius located well outside the Einstein ring of the lens. Shallow cusps, particularly the $\rho \propto r^{-1}$ cusps of the Hernquist and NFW models, are inconsistent with the data for any break radius. Lenses with shallow central cusps and break radii larger than the Einstein radius of the lens generically predict visible central images, and are ruled out by the absence of such images in well-defined lens samples (Rusin \& Ma 2000). Shallow cusps combined with a break radius smaller than the Einstein ring can demagnify the central image, but the monopole deflection curve (the rotation curve) becomes centrally peaked and is ruled out by the need for a flat deflection profile to fit the positions of the observed images. A massive central black hole can also demagnify central images (Mao, Witt \& Koopmans 2000), thereby weakening the limits on shallow cusps (or a central core radius) based on the absence of central images. In the case of $\mathrm{B} 1933+507$, the restrictions on shallow cusps are only partially due to the limits on the presence of central images, and when we eliminate the flux limits on the presence of a central image the restriction on the cusp exponent is only reduced to $\gamma \gtrsim 1.5$ from $\gamma \gtrsim 1$. The generality of the new developed cusped models yields a very robust prediction of the time delay for this system. In particular the lower limit obtained in the time delay computation for any cusped exponent $\gamma$ and any break radius (see Figure 9) would produce a strong constraint in the upper value of the Hubble constant provided an observational measurement of the time delay.

Acknowledgments: We thank the CASTLES collaboration (E.E. Falco, C.D. Impey, C.R. Keeton, C.S. Kochanek, J. Lehar, B.A. McLeod, J.A. Muñoz \& H.-W. Rix) for permission to include their APM 08279+5255 data. We also thank Joanne Cohn for providing us with her constraints for B $1933+507$ so that we could exactly match her models of B $1933+503$. CSK is supported by the Smithsonian Institution and NASA grant NAG5-9265. 


\section{REFERENCES}

Barkana, R., 1998, ApJ, 502, 531

Barkana, R., Lehár, J., Falco, E. E., Grogin, N. A., Keeton, C. R., \& Shapiro, I. I. 1999, ApJ, 520, 479

Bartelmann, M., 1996, A\&A, 313, 697

Biggs, A. D., Xanthopoulos, E., Browne, I. W. A., Koopmans, L. V. E. \& Fassnacht, C. D. 2000, MNRAS, 318, 73

Binney, J. \& Tremaine, S. 1987, Princeton, NJ, Princeton University Press, 1987, 747

Browne, I.W.A, Xanthopoulos, E., Biggs, A.D., \& Norbury, M., 1999, astro-ph/9909460

Chae, K., Turnshek, D. A. \& Khersonsky, V. K. 1998, ApJ, 495, 609

Chae, K., Khersonsky, V.K., \& Turnshek, D.A., 1998, ApJ, 506, 80

Chae, K. 1999, ApJ, 524, 582

Chen, G.H., Kochanek, C.S., \& Hewitt, J.N., 1995, ApJ, 447, 62

Cohn, J.D., Kochanek, C.S., McLeod, B.A. \& Keeton, C.R. 2001, ApJ submitted, astro-ph/0008390

Dehnen, W., 1993, MNRAS, 265, 250

de Vaucouleurs, G., 1948, Ann. Astrophys., 11, 247

Egami, E., Neugebauer, G., Soifer, B.T., Matthews, K., Ressler, M., Becklin, E.E., Murphy, T.W, Jr. \& Dale, D.A. 2000, ApJ, 535, 561

Ellithorpe, J.D., Kochanek, C.S., \& Hewitt, J.N., 1996, ApJ, 464, 556

Evans, N. W. \& Wilkinson, M. I. 1998, MNRAS, 296, 800

Faber, S. M. et al. 1997, AJ, 114, 1771

Fassnacht, C.D., Pearson, T.J., Readhead, A.C.S., Browne, I.W.A., Koopmans, L.V.E., Myers, S.T., \& Wilkinson, P.N., 1999, ApJ, 527, 498

Fukugita, M. \& Turner, E. L. 1991, MNRAS, 253, 99

Grogin, N. A. \& Narayan, R. 1996, ApJ, 464, 92

Hernquist, L. 1990, ApJ, 356, 359

Ibata, R.A., Lewis, G.F., Irwin, M.J., Lehár, J. \& Totten, E.J.,1999, AJ, 118, 1922 
Irwin, M.J., Ibata, R.A., Lewis, G.F. \& Totten, E.J. 1998, ApJ, 505, 529

Impey, C.D., Falco, E.E., Kochanek, C.S., Lehar, J., McLeod, B.A., Rix, H.-W., Peng, C.Y., \& Keeton, C.R., 1998, ApJ, 509, 551

Jaffe, W. 1983, MNRAS, 202, 995

Keeton, C.R., \& Kochanek, C.S., 1997, ApJ, 487, 42

Keeton, C. R., Kochanek, C. S. \& Seljak, U. 1997, ApJ, 482, 604

Keeton, C.R., \& Kochanek, C.S., 1998, ApJ, 495, 157

Keeton, C.R., Falco, E.E., Impey, C.D., Kochanek, C.S., Lehár, J., McLeod, B.A., Rix, H.-W.; Muñoz, J.A., \& Peng, C.Y. 2000, ApJ, 542, 74

Keeton, C.R., 2001, ApJ submitted, astro-ph/0102340

Keeton, C.R., \& Madau, P., 2001, ApJ in press, astro-ph/0101058

Kochanek, C.S., Blandford, R.D., Lawrence, C.R., \& Narayan, R., 1989, MNRAS, 238, 43

Kochanek, C.S., 1991, ApJ, 373, 354

Kochanek, C.S., \& Narayan, R., 1992, ApJ, 401, 461

Kochanek, C.S., 1995, ApJ, 445, 559

Kochanek, C.S., 1996, ApJ, 466, 638

Kochanek, C.S., Falco, E.E., Impey, C.D., Lehar, J., McLeod, B.A., Rix, H.-W., Keeton, C.R., Peng, C.Y., \& Muñoz, J.A., 2000, ApJ, 535, 692

Kochanek, C. S., Keeton, C. R., \& McLeod, B. A. 2001, ApJ, 547, 50

Koopmans, L. V. E. \& Fassnacht, C. D. 1999, ApJ, 527, 513

Kundic, T. et al. 1997, ApJ, 482, 75

Kuzmin, G. 1956, Astr. Zh., 33, 27

Lauer, T.R., 1985, ApJ, 292, 104L

Lehár, J., Falco, E.E., Kochanek, C.S., McLeod, B.A., Muñoz, J.A., Impey, C.D., Rix, H.-W., Keeton C.R. \& Peng, C.Y. 2000, ApJ, 536, 584

Ledoux, C., Theodore, B., Petitjean, P., Bremer, M.N., Lewis, G.F., Ibata, R.A., Irwin, M.J., \& Totten, E.J., 1988, A\&A, 339, 77L

Marlow, D.R., Browne, I.W.A., Jackson, P., \& Wilkinson, N., 1999, MNRAS, 305, 15 
Mao, S., Witt, H.J. \& Koopmans, L.V.E. 2001, MNRAS submitted, astro-ph/0007011

Mestel, L., 1963, MNRAS, 126, 553

Moore, B., Governato, F., Quinn, T., Stadel, J. \& Lake, G. 1998, ApJ, 499, L5

Nair, S., 1998, MNRAS, 301, 315

Navarro, J. F., Frenk, C. S. \& White, S. D. M. 1997, ApJ, 490, 493

Norbury et al. 2001, in preparation

Oscoz, A., Alcalde. D. Serra-Ricart, M., Mediavilla, E., Abajas, C., Barrena, R., Licandro, V. Motta, V. \& Muñoz, J.A. 2001, ApJ, in press, astro-ph/0102112

Rusin, D. \& Ma, C.-P., 2000, ApJL in press, astro-ph/0009079

Tremaine, S., Richstone, D. O., Byun, Y., Dressler, A., Faber, S. M., Grillmair, C., Kormendy, J. \& Lauer, T. R. 1994, AJ, 107, 634

Saha, P., \& Williams, L.L.R., 1997, MNRAS, 292, 148

Schechter, P.L., Bailyn, C.D., Barr, R., et al., 1997, ApJL, 475, 85

Schneider, P., Ehlers, J., \& Falco, E.E., 1992, Gravitational Lenses, (Springer: Berlin)

Schmidt, R., Webster, R. L., \& Lewis, G. F. 1998, MNRAS, 295, 488

Schramm, T. 1990, A\&A, 231, 19

Sykes, C.M., Browne, I.W.A., Jackson, N.J., et al. 1998, MNRAS, 301, 310

Wambsganss, J. \& Paczynski, B. 1994, AJ, 108, 1156

Wallington, S., Kochanek, C.S., \& Narayan, R., 1996, ApJ, 465, 64

Wyithe, J.S.B., Turner, E.L., \& Spergel, D.N., 2000, ApJ submitted, astro-ph/0007354

Zhao, H.S., 1996, MNRAS, 278, 488 
Table 1. APM 08279+5255 Photometry and Astrometry

\begin{tabular}{cccccccc}
\hline \hline ID & $\begin{array}{c}\Delta \alpha \\
\prime \prime\end{array}$ & $\begin{array}{c}\Delta \delta \\
\prime\end{array}$ & $\begin{array}{c}\text { Magnitude } \\
\text { V }\end{array}$ & $\begin{array}{c}\text { Color1 } \\
\text { V-I }\end{array}$ & $\begin{array}{c}\text { Color2 } \\
\text { V-J }\end{array}$ & $\begin{array}{c}\text { Color3 } \\
\text { V-H }\end{array}$ & $\begin{array}{c}\text { Color4 } \\
\text { V-K }\end{array}$ \\
\hline A & $\equiv 0$ & $\equiv 0$ & $16.96 \pm 0.03$ & $1.71 \pm 0.06$ & $3.51 \pm 0.03$ & $3.86 \pm 0.03$ & $4.72 \pm 0.04$ \\
$\mathrm{~B}$ & $-0.199 \pm 0.003$ & $-0.327 \pm 0.003$ & $17.74 \pm 0.03$ & $2.10 \pm 0.04$ & $3.99 \pm 0.03$ & $4.37 \pm 0.03$ & $5.23 \pm 0.03$ \\
$\mathrm{C}$ & $-0.100 \pm 0.003$ & $-0.105 \pm 0.003$ & $18.70 \pm 0.07$ & $1.77 \pm 0.09$ & $3.46 \pm 0.07$ & $3.96 \pm 0.07$ & $4.67 \pm 0.14$ \\
\hline
\end{tabular}

Note. - The infrared images (H, J and K bands) were obtained by Ibata et al. (1999) with the NIC1 camera at the HST. The optical images were acquired by the CASTLES collaboration.

Table 2. APM 08279+5255 Lens Models

\begin{tabular}{lcccccccccc}
\hline \hline Model & $\Delta \alpha\left(^{\prime \prime}\right)$ & $\Delta \delta\left(^{\prime \prime}\right)$ & $b^{*}\left({ }^{\prime \prime}\right)$ & $a^{\dagger}\left({ }^{\prime \prime}\right)$ & $\epsilon(1-b / a)$ & $\theta_{\epsilon}\left(^{\circ}\right)$ & $h \Delta t_{A B}^{\ddagger}$ & $h \Delta t_{C B}^{\ddagger}$ & $\mu_{T}$ & $\chi^{2}$ \\
\hline SIE & -0.117 & -0.125 & 0.42 & 0.16 & 0.019 & 79 & 0.49 & 0.63 & 125 & 0 \\
Exp. disk & -0.116 & -0.124 & 1.38 & 0.37 & 0.022 & 80 & 0.56 & 0.72 & 113 & 0 \\
Cusp $(\gamma=0.1)$ & -0.117 & -0.126 & 0.82 & 0.28 & 0.029 & 78 & 0.74 & 0.96 & 62 & 0 \\
Cusp $(\gamma=0.2)$ & -0.117 & -0.125 & 0.66 & 0.39 & 0.020 & 76 & 0.51 & 0.66 & 129 & 0 \\
Cusp $(\gamma=0.2)$ & -0.117 & -0.125 & 0.54 & 1.02 & 0.005 & 73 & 0.13 & 0.17 & 1814 & 0 \\
Cusp $(\gamma=0.3)$ & -0.117 & -0.123 & 0.55 & 0.54 & 0.017 & 74 & 0.42 & 0.52 & 210 & 0.1 \\
Cusp $(\gamma=0.4)$ & -0.116 & -0.120 & 0.51 & 0.50 & 0.025 & 75 & 0.60 & 0.71 & 118 & 0.7 \\
\hline
\end{tabular}

Note. -

* In the case of the cusped models it corresponds to the dimensionless normalization $\kappa_{0}$ used in the text. $\dagger$ "a" is the core radius in the SIE model, the scale radius for the exponential disk, and the break radius in the cusped model.

${ }^{\ddagger}$ For the time delay computation we used $z_{l}=1.18$, corresponding to a strong MgII absorption system, for the unknown redshift of the lens. The time delay is given in days in a flat $\left(\Omega_{0}=0.3\right)$ cosmology with $H_{0}=100 h \mathrm{~km} \mathrm{~s}^{-1} \mathrm{Mpc}^{-1}$. 
Table 3. B 1933+503 Lens Models

\begin{tabular}{lcccccccccc}
\hline \hline Model & $\Delta \alpha\left(^{\prime \prime}\right)$ & $\Delta \delta\left(^{\prime \prime}\right)$ & $k_{0}$ & $a\left(^{\prime \prime}\right)$ & $\epsilon(1-b / a)$ & $\theta_{\epsilon}\left({ }^{\circ}\right)$ & $\gamma_{s}($ shear $)$ & $\theta_{\gamma_{s}}\left({ }^{\circ}\right)$ & $h \Delta t_{\text {max }}^{\dagger}$ & $\chi^{2}$ \\
\hline Cusp $(\gamma=1.1)$ & 0.033 & -0.029 & 0.926 & 0.44 & 0.331 & -41.6 & 0.174 & -47.6 & 15.4 & 16.2 \\
Cusp $(\gamma=1.3)$ & 0.035 & -0.031 & 0.569 & 0.59 & 0.349 & -41.7 & 0.147 & -48.3 & 15.0 & 13.4 \\
Cusp $(\gamma=1.5)$ & 0.035 & -0.032 & 0.343 & 0.79 & 0.363 & -41.5 & 0.125 & -49.3 & 14.4 & 12.1 \\
Cusp $(\gamma=1.7)$ & 0.035 & -0.033 & 0.168 & 1.27 & 0.388 & -42.1 & 0.095 & -50.5 & 13.7 & 11.4 \\
Cusp $(\gamma=1.8)$ & 0.035 & -0.036 & 0.021 & $\equiv 10$ & 0.406 & -42.3 & 0.022 & -73.9 & 10.9 & 11.0 \\
Cusp $(\gamma=1.85)$ & 0.034 & -0.033 & 0.018 & $\equiv 10$ & 0.407 & -42.4 & 0.031 & -63.6 & 11.3 & 10.8 \\
Cusp $(\gamma=1.9)$ & 0.034 & -0.032 & 0.015 & $\equiv 10$ & 0.407 & -42.5 & 0.041 & -57.7 & 11.6 & 11.0 \\
Cusp $(\gamma=2.0)$ & 0.032 & -0.031 & 0.011 & $\equiv 10$ & 0.413 & -42.7 & 0.061 & -52.6 & 12.3 & 12.1 \\
Cusp $(\gamma=2.1)$ & 0.031 & -0.030 & 0.008 & $\equiv 10$ & 0.415 & -42.6 & 0.089 & -50.1 & 13.2 & 14.0 \\
\hline
\end{tabular}

Note. - The best models for $\gamma \gtrsim 1.8$ have fit statistics that decline monotonically for increasing break radii $a$. We show models with the break radius fixed to $a=10^{\prime \prime}$ for these cases. The sudden jump in the break radius leads to the discontinuity in the parameter trends between the $\gamma=1.7$ and $\gamma=1.8$ models.

${ }^{\dagger}$ Longest time delay between the four images of the compact radio-core (it corresponds to images 1 and 6 , see figure 5) given in days in a flat $\left(\Omega_{0}=0.3\right)$ cosmology with $H_{0}=100 h \mathrm{~km} \mathrm{~s}^{-1} \mathrm{Mpc}^{-1}$. 